\title{
Endoscopic posterior interhemispheric complete corpus callosotomy
}

\author{
Sandeep Sood, MD, ${ }^{1}$ Eishi Asano, MD, PhD, ${ }^{2}$ Deniz Altinok, MD, ${ }^{3}$ and Aimee Luat, MD $^{2}$ \\ Departments of ${ }^{1}$ Pediatric Neurosurgery, ${ }^{2}$ Pediatric Neurology, and ${ }^{3}$ Pediatric Radiology, Wayne State University, Children's \\ Hospital of Michigan, Detroit, Michigan
}

\begin{abstract}
Traditionally corpus callosotomy is done through a craniotomy centered at the coronal suture, with the aid of a microscope. This involves dissecting through the interhemispheric fissure below the falx to reach the corpus callosum. The authors describe a posterior interhemispheric approach to complete corpus callosotomy with an endoscope, which bypasses the need to perform interhemispheric dissection because the falx is generally close to the corpus callosum in this region.

http://thejns.org/doi/abs/10.3171/2016.6.PEDS16131
\end{abstract}

KEY WORDS corpus callosotomy; minimally invasive surgery; endoscopic surgery; intractable seizures; drop attacks; epilepsy; technique

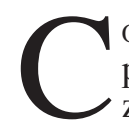
ORPus callosotomy is an established disconnective procedure used for patients with drug-resistant seizures, notably drop attacks or astatic seizures. The procedure is done through a craniotomy either centered on the coronal suture or precoronally, with the aid of a microscope-or, more recently, using an endoscope. ${ }^{3}$ In either case, it involves dissecting the 2 hemispheres below the level of the falx to reach the corpus callosum surface. It is our experience that often patients with brain malformations may have a deficient falx anteriorly or a high-riding falx. This makes the dissection more difficult because the gyri of each hemisphere may interdigitate across the midline into the other hemisphere. In addition, patients with frequent drop attacks often have more marked adhesions due to repeated injury related to the falls. In this paper we describe a posterior interhemispheric endoscopic approach to complete corpus callosotomy that bypasses the need for interhemispheric dissection due to the proximity of the falx and the corpus callosum posteriorly.

\section{Methods}

Two patients who were 13 and 19 years of age (Cases 1 and 2, respectively) had a diagnosis of Lennox-Gastaut syndrome and suffered from drug-resistant multiple seizure types, including tonic and astatic seizures. Neither patient showed evidence of unifocal epilepsy. The patient in Case 1 had a diagnosis of bilateral lissencephaly, and the one in Case 2 was found to have an SCNIA genetic variant of unknown significance. Scalp electroencephalography (EEG) showed multifocal interictal spike-wave discharges as well as diffuse repetitive spike-wave discharges at $<3 \mathrm{~Hz}$; ictal EEG showed a diffuse onset. After intense discussion of the pros and cons of each treatment option, the families of the patients decided to pursue complete corpus callosotomy. Both patients had a reasonably high-riding falx (Fig. 1), and therefore consideration was given to the described approach. Preoperative MRI scans were obtained for neuronavigation and an MR venogram was done for approach planning in both patients.

Patients were positioned prone in a $30^{\circ}$ reverse Trendelenburg position with the head secured in pin fixation and with a $20^{\circ}-30^{\circ}$ neck flexion. Both patients underwent registration of the neuronavigation system for intraoperative guidance and preoperative planning. The trajectory was planned to perform the craniotomy away from the bridging veins in the posterior parietooccipital region, so that the rostrum of the corpus callosum could be accessed 


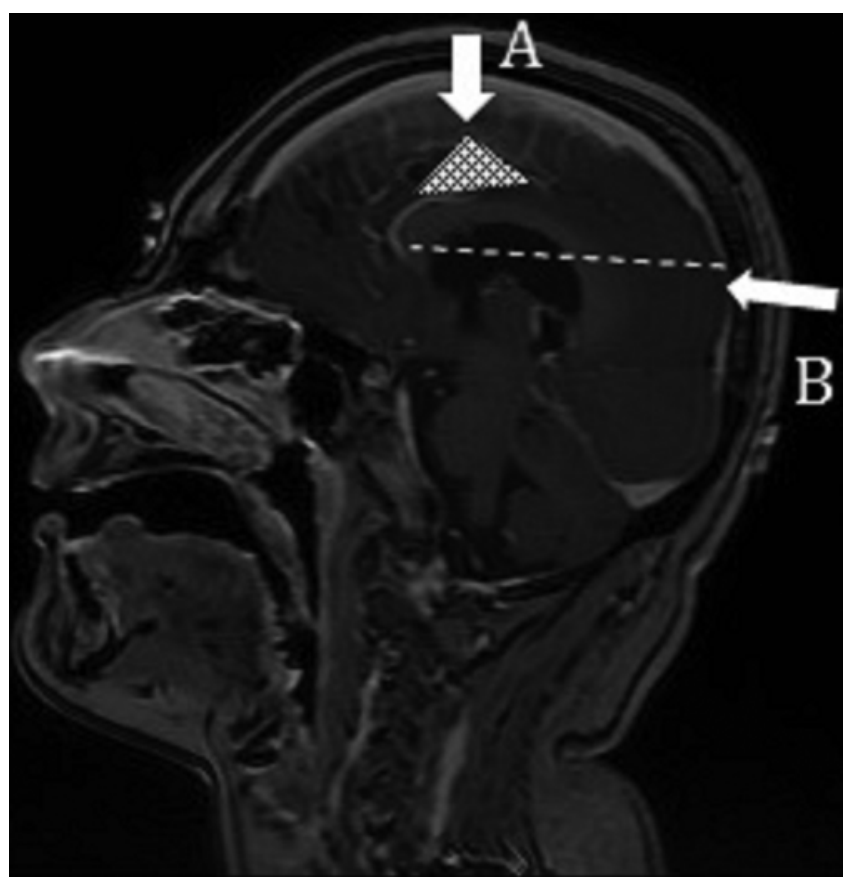

FIG. 1. Neuroimage showing the standard coronal approach (arrow A), which involves dissection of the interhemispheric fissure from the lower end of the falx to the corpus callosum along the shaded area. The posterior interhemispheric endoscopic approach (arrow $B$ ) is made along the long axis of the corpus callosum (dotted line) and does not require interhemispheric dissection.

over the curve of the fornix, as shown in Fig. 1 (arrow B). After appropriate surgical prepping and draping, an incision was made approximately $3 \mathrm{~cm}$ in length and approximately $1-1.5 \mathrm{~cm}$ lateral to the midline (Fig. $2 \mathrm{~B}$ and $\mathrm{C}$ ). A D-shaped craniotomy was made with the base situated medially. The dura mater was opened with the flap based medially. Intravenous mannitol (20\%, $1 \mathrm{~g} / \mathrm{kg}$ body weight) was used to relax the brain in both patients. In addition, in 1 patient, a ventricular catheter was placed under neuronavigation guidance into the occipital horn to drain CSF and to achieve brain relaxation.

After adequate brain relaxation, a single retractor was placed to the depth of the corpus callosum, creating a $1-\mathrm{cm}$ corridor, gently retracting the brain laterally. Subsequently an endoscope held in the left hand, with mounted suction, was introduced in the field and, with the Cavitron ultrasonic surgical aspirator (CUSA) held in the right hand, the corpus callosum was removed sequentially in a posterior-to-anterior direction. This was done by starting at the site of exposure of the corpus callosum, which generally corresponds to the junction of the anterior twothirds and posterior one-third, strictly staying in the midline, and following the groove of the septum pellucidum inferiorly and the interhemispheric fissure superiorly (Fig. $3 \mathrm{~A}-\mathrm{F}$ and Videos 1 and 2).

VIDEO 1. Clip showing 2D endoscopic view: endoscopic posterior interhemispheric complete corpus callosotomy. $\mathrm{Cc}=$ corpus callosum; $\mathrm{Fx}=$ fornix; $\mathrm{Ge}=$ genu; $\mathrm{Hc}=$ hippocampal commissure; $\mathrm{IHF}=$ interhemispheric fissure; $\mathrm{R}=$ rostrum; $\mathrm{Sp}=$ septum pellucidum; $\mathrm{Spl}$ $=$ splenium. Copyright Sandeep Sood. Published with permission. Click here to view.

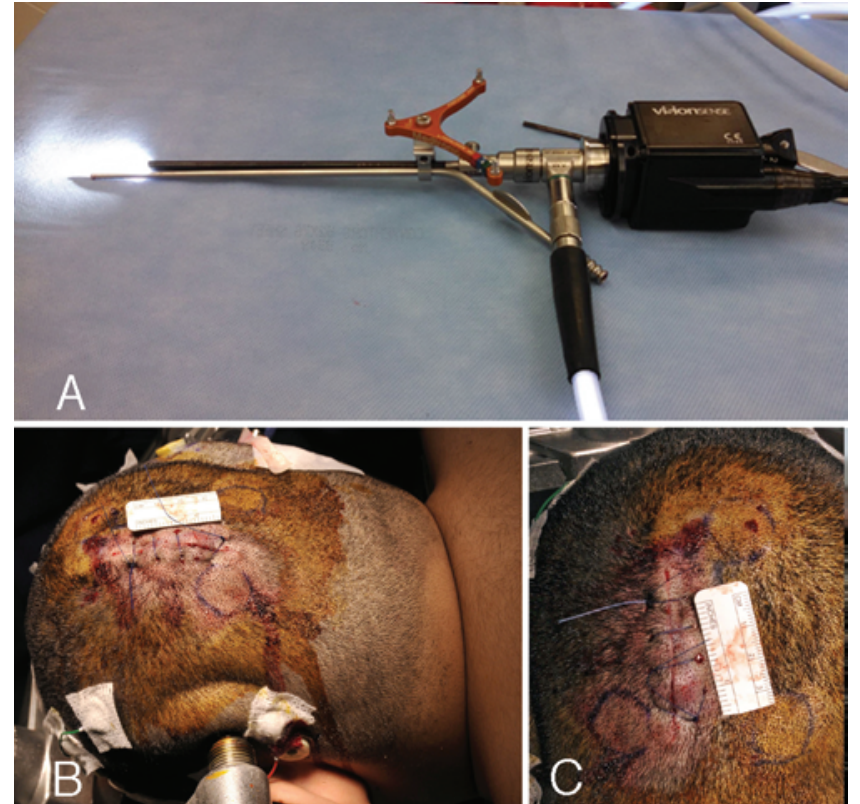

FIG. 2. A: Endoscope with the attached suction and neuronavigation guide is held in the surgeon's left hand, and bimanual dissection is performed with a second instrument held in the surgeon's right hand. B and C: Photographs showing the position and the incision required to perform complete corpus callosotomy using the endoscopic method via the parietooccipital approach with the patient in the prone position. Figure is available in color online only.

VIDEO 2. Clip showing 3D endoscopic view stereo pair video to be viewed approximately 18 inches from the screen. Fuse the right and the left images by viewing cross-eyed; the center image appears in 3D: endoscopic posterior interhemispheric complete corpus callosotomy. Ant = anterior; $\mathrm{Cc}=$ corpus callosum; $\mathrm{IHF}=$ interhemispheric fissure; Post = posterior; $R=$ rostrum; $S p=$ septum pellucidum; $S p l$ = splenium. Copyright Sandeep Sood. Published with permission. Click here to view.

The CUSA microtip was used at settings with aspiration 20-30, vibration 30-40, and tissue setting at low. Because the corpus callosum removal is done essentially intracallosally, the anterior cerebral arteries may not be visualized and do not need to be dissected. They were above the corpus callosum, away from the field of dissection. Anteriorly, the leaves of the septum pellucidum were separated to reach the rostrum over the hump of the fornix. In 1 patient, because the septum was adherent, it was necessary to enter the ventricle on 1 side. The rostrum was approached from within the ventricle, following the curve of the corpus callosum in this patient. Hemostasis from the edges was easily controlled with a bipolar device under endoscopic vision.

At this point our attention was directed to the posterior part of the corpus callosum. This was removed with the CUSA, following the curve of the arachnoid that covered the vein of Galen onto the internal cerebral veins. The hippocampal commissure was split in the midline and this dissection was carried anteriorly, and the septum was reached at the completion of the disconnection.

In the first patient, an external ventricular drain was left in place for 24 hours after surgery, but in the second patient no drains were used. 

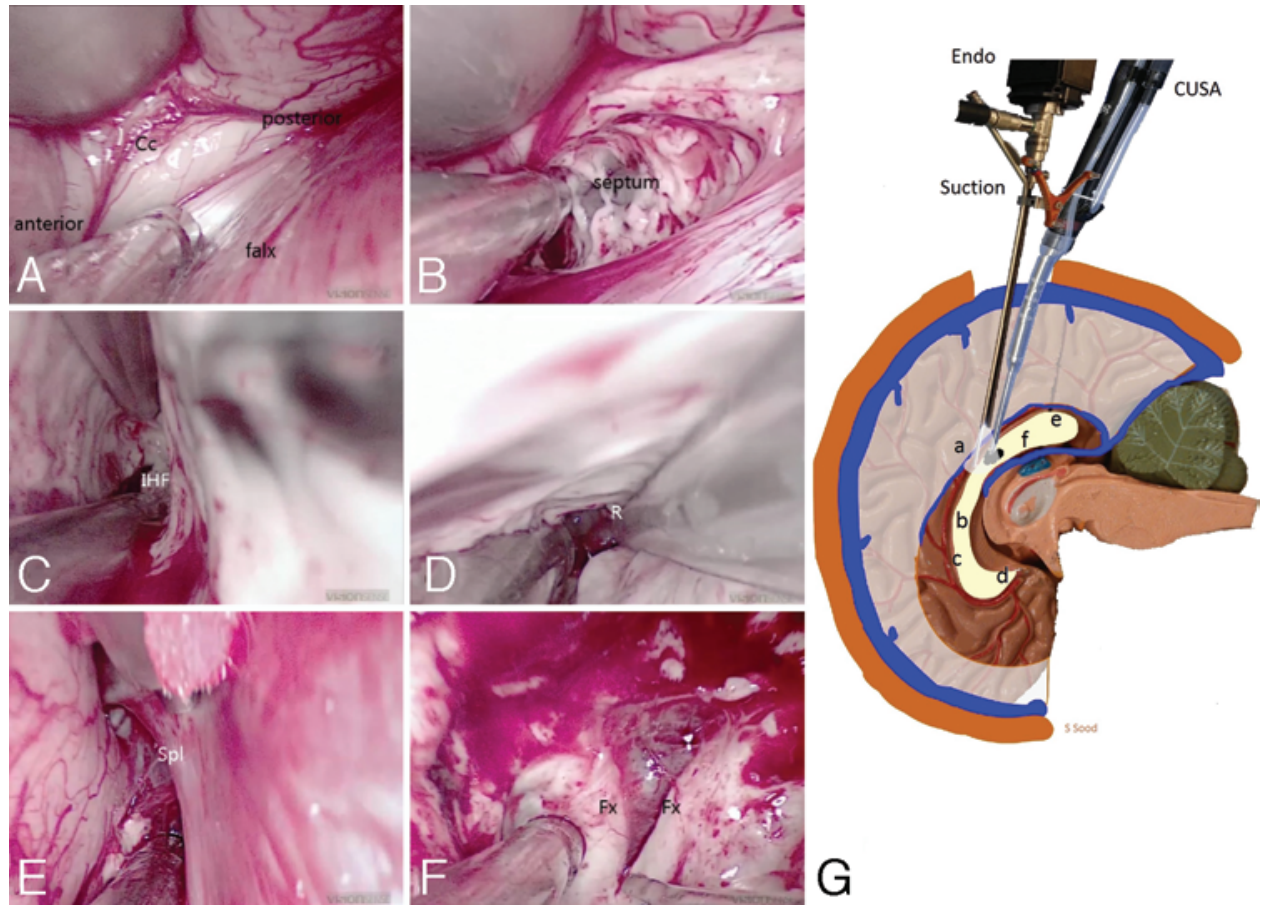

FIG. 3. A-F: Endoscopic views along the corresponding trajectories shown in panel $\mathbf{G}$ (labels a-f). Cc = corpus callosum; Endo = endoscope; Fx = fornix; IHF = interhemispheric fissure; R = rostrum; Spl = splenium. Panel G: Copyright Sandeep Sood. Published with permission. Figure is available in color online only.

\section{Results}

Both patients tolerated the positioning well. The endoscope time was 2 hours in one patient and 1.5 hours in the other. The blood loss was insignificant and neither of the patients required blood transfusions. Both patients achieved intraoperative EEG desynchronization at the completion of corpus callosotomy. Hospital stay was 4 days in one and 5 days in the other patient. Postoperative MRI with diffusion tensor imaging showed complete disconnection of the corpus callosum (Fig. 4).

\section{Discussion}

In patients with a high-riding falx, a substantial amount of interhemispheric dissection may be required to get to the corpus callosum from the traditional coronal craniotomy approach (Fig. 1). Because the falx is close to the corpus callosum posteriorly, it seems reasonable to consider a posterior approach in these patients. However, the operating distance from the parietooccipital brain surface to the genu is generally $9-12 \mathrm{~cm}$. At this distance, surgery with an operating microscope becomes difficult and unsafe. Obscuring of vision from the instruments as well as the lighting and depth of focus become a challenge.

In contrast, the depth to which the surgery can be performed with an endoscope is essentially only limited by the length of the endoscope. The drawbacks of conventional use of endoscopy with instrumentation through the side channel include the limited ability to dissect or control bleeding. Use of an endoscope on a holder through a ventricular port would undesirably require a larger craniotomy to allow space for placement of the endoscope and for the instruments that the surgeon uses in the right and left hands. ${ }^{1}$ In addition, if the field of surgery is larger than the view through the endoscope, a surgeon might be required to repeatedly reposition the endoscope to the desired field of surgery. Furthermore, this approach, the so-called 3-handed technique, results in crowding of instruments. Finally, in this method, when the instruments are introduced into the field they cannot be seen until they reach beyond the tip of the endoscope.

The senior author (S.S.) uses a novel endoscopic surgery system, which does not suffer from the aforementioned limitations. By attaching a rigid suction apparatus to the endoscope (Fig. 2A) the surgeon is able to use the suction in the left hand and a second instrument in the right hand in a standard microsurgical fashion. Because the endoscope is attached to the suction it moves with the suction to the site of surgical dissection, eliminating the need for repositioning. We have found that this combination for bimanual dissection was effective for corpus callosotomy through the precoronal craniotomy (anterior approach), ${ }^{3}$ for hemispherotomy, ${ }^{3}$ and for resection of deep thalamic ${ }^{4}$ and pineal tumors. ${ }^{2}$ Using this combination of a suction device attached to an endoscope, the depth to which surgery can be performed through a microcraniotomy $(2-3 \mathrm{~cm})$ can be extended beyond the limit of the microscope. A stereotactic attachment further aids in surgical navigation. We have used both 2D and 3D endoscopes for this procedure. Using a 2D endoscope at a depth of more than $5 \mathrm{~cm}$ becomes difficult, and frequent reassessment of depth with neuronavigation is tedious. We have found that using a $3 \mathrm{D}$ endoscope (such as VisionSense) is more surgeon friendly. 

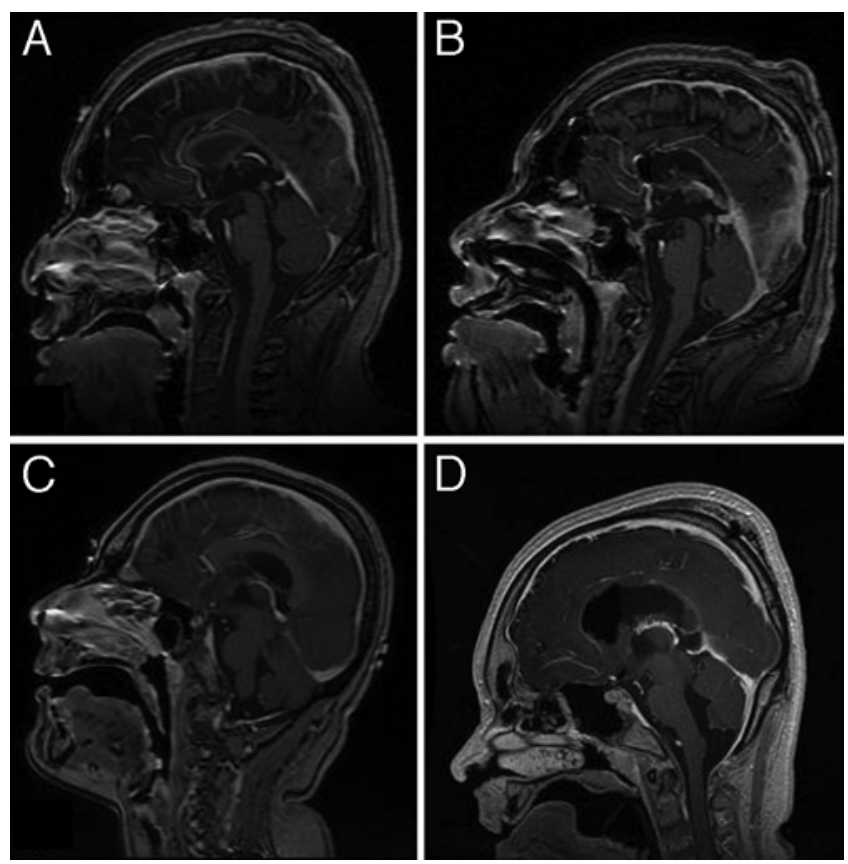

FIG. 4. Preoperative (A and $C$ ) and postoperative (B and D) sagittal $M R$ images demonstrating complete corpus callosotomy in Case 1 ( $A$ and $B$ ) and Case 2 (C and D). A postoperative MRI study obtained in the second patient shows some layering of blood in the cavity of the resected splenium.

Use of neuronavigation is important in planning the surgery. The site of craniotomy is critical to obtain the appropriate trajectory to the rostrum by splitting the septum pellucidum. In one of our patients, in whom the septum was very adherent, the rostrum was easily accessed transventricularly. Endoscopic corpus callosotomy aims at minimizing the size of incision and craniotomy. The posterior approach as described above would be rather difficult to do with a microscope and, to our knowledge, has not been attempted in the past. It is, however, feasible and safe with our endoscopic system described above. It has clear advantages over the anterior approach. First, minimal interhemispheric dissection is required because the falx is closely approximated to the corpus callosum posteriorly. Second, the anterior cerebral arteries are not exposed because the entire procedure is done essentially intracallosally, so the risk of injury to the vessels or subsequent spasm are minimized. The ability to use the endoscope with attached suction bimanually allows the use of bipolar cautery in the right hand to control any inadvertent bleeding, much as in microscopic surgery. Finally, the procedure is performed from the parietooccipital region, and the small amount of retraction done to develop the corridor for introducing the endoscope does not risk the motor strip; i.e., the leg area or the supplementary motor strip. Such a risk is not negligible with the frontal microscopic approach.

\section{Conclusions}

At our institution, complete corpus callosotomy is offered as a 1-stage procedure to patients with intractable drop attacks who have significant developmental delay and are unable to read. In these patients intraoperative EEG may be used as a guide to the extent of resection. Both patients in this study fulfilled these criteria and had complete corpus callosotomy to achieve desynchronization on intraoperative scalp EEG. However, it is possible to do only an anterior two-thirds callosotomy via this approach in patients who may have a higher cognitive function than the individuals in this paper. Furthermore, entry into the third ventricle between the 2 internal cerebral veins would permit a more extensive midline disconnection of the posterior and anterior commissures if needed.

\section{References}

1. Harris AE, Hadjipanayis CG, Lunsford LD, Lunsford AK, Kassam AB: Microsurgical removal of intraventricular lesions using endoscopic visualization and stereotactic guidance. Neurosurgery 56 (1 Suppl):125-132, 2005

2. Sood S, Hoeprich M, Ham SD: Pure endoscopic removal of pineal region tumors. Childs Nerv Syst 27:1489-1492, 2011

3. Sood S, Marupudi NI, Asano E, Haridas A, Ham SD: Endoscopic corpus callosotomy and hemispherotomy. J Neurosurg Pediatr 16:681-686, 2015

4. Sood S, Nundkumar N, Ham SD: Interhemispheric endoscopic resection of large intraventricular and thalamic tumors. J Neurosurg Pediatr 7:596-599, 2011

\section{Disclosures}

The authors report no conflict of interest concerning the materials or methods used in this study or the findings specified in this paper.

\section{Author Contributions}

Conception and design: Sood. Acquisition of data: Sood, Asano. Analysis and interpretation of data: all authors. Drafting the article: all authors. Critically revising the article: Sood. Reviewed submitted version of manuscript: Sood, Asano, Luat. Approved the final version of the manuscript on behalf of all authors: Sood.

\section{Supplemental Information \\ Videos}

Video 1. https://vimeo.com/174215289.

Video 2. https://vimeo.com/174215385.

\section{Previous Presentations}

This paper was presented in part at the 39th annual meeting of the American Society of Pediatric Neurosurgery, held in Aruba, on January 31-February 5, 2016.

\section{Correspondence}

Sandeep Sood, Department of Neurosurgery, Wayne State University School of Medicine, Children's Hospital of Michigan, 3901 Beaubien, 2nd Fl., Detroit, MI 48201. email: ssood@med. wayne.edu. 